\title{
Hybrid Fixation for Paediatric Femoral Supracondylar Fracture during Circular External Fixation of the Lower Limb
}

\author{
Yoshinobu Oka ${ }^{1}$, Wook-Cheol Kim², Takashi Yoshida ${ }^{3}$, Masashi Nakase ${ }^{4}$, Yoshihiro Kotoura ${ }^{5}$, Atsushi Nishida ${ }^{6}$, Hiroaki Wada ${ }^{7}$, \\ Toshiharu Shirai $^{8}$, Kenji Takahashi ${ }^{9}$
}

\begin{abstract}
Aim: To describe the novel hybrid fixation technique for paediatric femoral supracondylar fracture during circular external fixation of the lower limb. Background: The llizarov external fixator is commonly used for various orthopaedic conditions. Difficulties associated with external fixation have previously been described. A fall while using a circular external fixator can cause ipsilateral fracture. Such fractures are ideally treated conservatively, but it is difficult to fix the frame itself. No study has reported the treatment of paediatric femoral supracondylar fracture during circular external fixation. Herein, we describe a novel hybrid fixation technique that was successfully used to treat paediatric femoral supracondylar fracture in three paediatric patients with circular external fixators.

Technique: The fracture was manually manipulated and reduced by slight hyperextension of the lower extremity under general anaesthesia. After confirmation of good reduction, a stockinette, a cast padding, and a thin core cast were applied to the ipsilateral thigh. The hinge parts were attached to the medial and lateral sides of the proximal ring. The rods were connected to the medial and lateral hinges, and the half ring was connected to the ventral side of the proximal end. Under fluoroscopic confirmation, the thin core cast of the thigh and rods were connected by cast rolled in a figure-of-eight manner. The hinges were locked with the knee joint slightly flexed.

Conclusion: The minimally invasive hybrid fixation technique enables conservative treatment of paediatric femoral supracondylar fracture during circular external fixation of the lower limb with no complications, and early exercise and recovery.

Clinical significance: This novel hybrid fixation technique will be an effective method for paediatric femoral supracondylar fracture in patients with a circular external fixator.

Keywords: Children, Circular external fixation, Femur fracture.

Strategies in Trauma and Limb Reconstruction (2020): 10.5005/jp-journals-10080-1454
\end{abstract}

\section{BACKGROUND}

Circular external fixators, such as the llizarov external fixator, are used for treating open fractures, ${ }^{1}$ limb lengthening, ${ }^{2}$ and reconstruction after massive bone loss. ${ }^{3}$ Complications associated with limb lengthening have been reported previously. ${ }^{4}$ Common issues are muscle contractures, joint subluxation, neurological and vascular injuries, premature consolidation of the lengthened bone, pin site problems, and hardware failure. In addition, a fracture is a serious complication associated with the frame and after frame removal. The incidence of fracture, associated with a previous pin track or elsewhere, is as high as $5-18 \% .^{5-7}$ However, an ipsilateral fracture during circular external fixation is rare, with an ipsilateral tibial stress fracture reportedly occurring in approximately $4 \%$ of adults when treated for Charcot foot. ${ }^{8}$ One study reported that fracture at a distant site after external fixation only occurred in 3 of 17 cases. ${ }^{6}$ The incidence or treatment of paediatric femoral supracondylar fracture during treatment with a circular external fixator on the tibia has not been reported previously.

In long-term circular external fixation for reconstruction of the tibia, tolerable weight-bearing is recommended to encourage bone maturation. ${ }^{9}$ However, this can lead to femoral supracondylar fracture if the patient falls during weight-bearing. In such cases, the treatment in adults is rigid internal fixation, such as locking plate fixation, ${ }^{10}$ because this achieves fracture healing in correct alignment and enables an early return to functional activity. In contrast, children with an open physis need other options for fracture fixation. Conservative treatment via cast immobilisation is difficult because of the presence of the circular external fixator.
${ }^{1}$ Department of Pediatric Orthopaedics, Kyoto Prefectural University of Medicine, Kyoto, Japan

${ }^{2}$ Department of Pediatric Orthopaedics and Ilizarov Center, Uji Takeda Hospital, Kyoto, Japan

${ }^{3-9}$ Department of Orthopaedics, Kyoto Prefetural University of Medicine, Kyoto, Japan

Corresponding Author: Yoshinobu Oka, Department of Pediatric Orthopaedics, Kyoto Prefectural University of Medicine, Kyoto, Japan, Phone: +91 81752515549, e-mail: y-oka@koto.kpu-m.ac.jp

How to cite this article: Oka Y, Kim W-C, Yoshida T, et al. Hybrid Fixation for Paediatric Femoral Supracondylar Fracture during Circular External Fixation of the Lower Limb. Strategies Trauma Limb Reconstr 2020;15(3):179-183.

Source of support: Nil

Conflict of interest: None

Other options include surgical procedures, such as closed reduction and percutaneous K-wire fixation, ${ }^{11}$ titanium elastic intramedullary nailing, ${ }^{12}$ extension of the external fixation, ${ }^{13}$ and open reduction and plate fixation. ${ }^{14}$ However, as healing of fractures around the metaphysis is relatively fast and predictable, noninvasive treatment is recommended when possible.

We report our experience with a novel hybrid fixation technique for femoral supracondylar fractures in paediatric patients who have a circular external fixator on the lower limb. 


\section{TECHNIQUe}

Hospital records were reviewed to identify paediatric patients with ipsilateral fractures of the femur during circular external fixation of the tibia (three patients, four femurs: Table 1). The mean age was 6 years and 7 months and the patient demographics are listed in Table 1. There were two patients who had fixation by an Ilizarov circular fixator for congenital pseudoarthrosis of the tibia and the third for bone transport using a Taylor Spatial Frame after resection of an Ewing's sarcoma. In all cases, the femoral supracondylar fracture was of a greenstick-type and flexed.

\section{Operative Technique}

All operations were performed under general anaesthesia on a radiolucent table. The fracture was manipulated manually and reduced using mild hyperextension of the lower extremity. After fluoroscopic confirmation of good reduction on the anteroposterior (AP) and lateral views, a stockinette, a cast padding, and a thin cast using one cast roll was applied to the thigh. The hinge parts were then applied on the medial and lateral sides of the proximal ring. The positions of the hinges were aligned along the transepicondylar axis of the knee. Rods were then connected to the medial and lateral hinges, and a half ring connected to the ventral side of the proximal end to achieve good stability (Fig. 1). After fluoroscopic confirmation, the thin core cast of the thigh and rods were bound using two or three rolls of cast rolled in a figure-of-eight manner. The knee joint was fixed in a slightly flexed position, and the hinges were locked (Fig. 2). Postoperatively, active hip range of motion exercises were carried out as tolerated. Partial weight-bearing was permitted after confirmation of callus formation on plain radiography. At 2 or 3 weeks postoperatively, the hinges were loosened and knee range of motion exercise was started.

At confirmation of fracture union by radiographs, the cast, rods, and hinges were removed. The mean additional femoral fixation period was 35 days (range 21-44 days). All three patients quickly recovered their ability to perform activities of daily living, and there
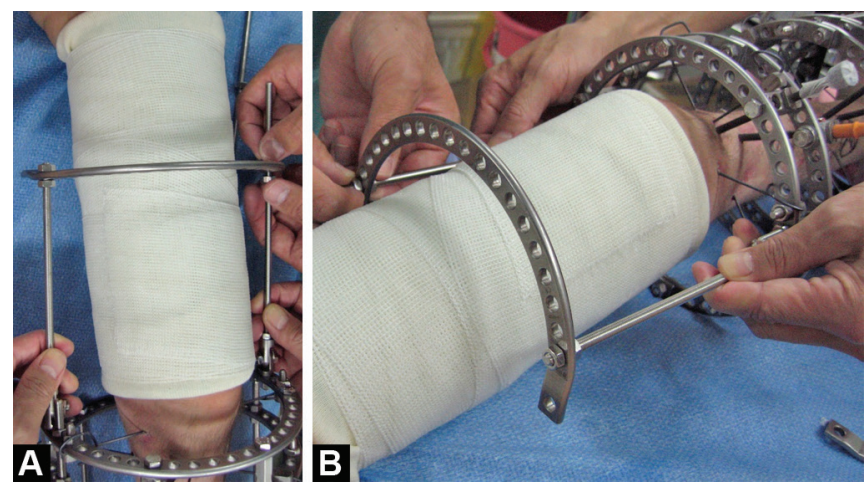

Figs $1 \mathrm{~A}$ and $\mathrm{B}$ : A thin core cast is applied to the thigh. The hinges, rods, and ventral side half ring are connected to the proximal ring were no complications, such as loss of reduction, malunion (final anatomical posterior distal femoral angle (aPDFA): average 88.0 range 87.3-88.4). The final knee range of motion was $0^{\circ}$ in extension and $150^{\circ}$ in flexion in all cases.

\section{Case Description}

Case 1 was a 9 years and 10 months-old boy with a left congenital pseudoarthrosis of the tibia. We performed a four-in-one procedure, with autologous bone graft and proximal limb lengthening. Five months postoperatively, he fell and incurred a left femoral supracondylar greenstick fracture. Under general anaesthesia, closed reduction and stabilisation was performed using the hybrid fixation method (Fig. 3). Callus formation around the fracture site was confirmed at 3 weeks post-operatively, and the medial and lateral hinges were loosened to allow range of motion exercise. Partial weight-bearing was encouraged as tolerated. The cast and rods were removed at 34 days postoperatively. Complete union was achieved with no deformity.

The knee range of motion was $0^{\circ}$ to $150^{\circ}$ at final examination (Fig. 4).

Case 2 was a 9 years and 3 months-old boy with Ewing's sarcoma of the right distal tibia. He was first seen in our department aged 8 years and 1 month-old and underwent preoperative chemotherapy followed by wide resection. Reconstruction was performed by bone transport using a Taylor Spatial Frame. He fell and incurred a right femoral supracondylar fracture (Fig. 5). This fracture was reduced with slight hyperextension of the lower leg and stabilised via hybrid fixation. At 3 weeks postoperatively, the cast and rods were removed, and exercise was started. His range of motion of the knee was $0^{\circ}$ in extension and $150^{\circ}$ in flexion at final follow-up, with no evidence of malunion (Fig. 6).

\section{Discussion}

Although patients can fall while ambulating in a circular external fixator, sustaining an ipsilateral fracture is rare. Ipsilateral tibial stress
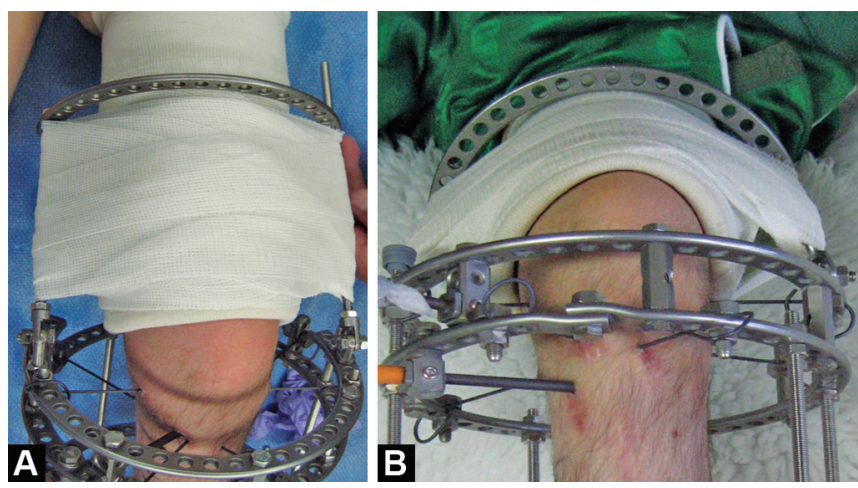

Figs $2 \mathrm{~A}$ and B: The core cast and rods are connected by a cast rolled in a figure-of-eight fashion. The hinges are locked with the knee joint slightly flexed

Table 1: Patient demographics

\begin{tabular}{|c|c|c|c|c|c|c|}
\hline Case & Age & Sex & Laterality & Diagnosis & Fixator & Duration (days) \\
\hline 1 & $3 Y+10$ & $M$ & $\mathrm{~L}$ & CPT & Ilizarov & 44 \\
\hline 1 & $9 Y+10$ & $\mathrm{M}$ & $\mathrm{L}$ & СРT & Ilizarov & 34 \\
\hline 2 & $9 Y+3$ & $\mathrm{M}$ & $\mathrm{R}$ & Ewing's sarcoma & $\begin{array}{l}\text { Taylor spatial } \\
\text { frame }\end{array}$ & 21 \\
\hline 3 & $3 Y+5$ & $\mathrm{~F}$ & $\mathrm{~L}$ & СРT & Ilizarov & 41 \\
\hline
\end{tabular}

CPT, congenital pseudoarthrosis of the tibia 

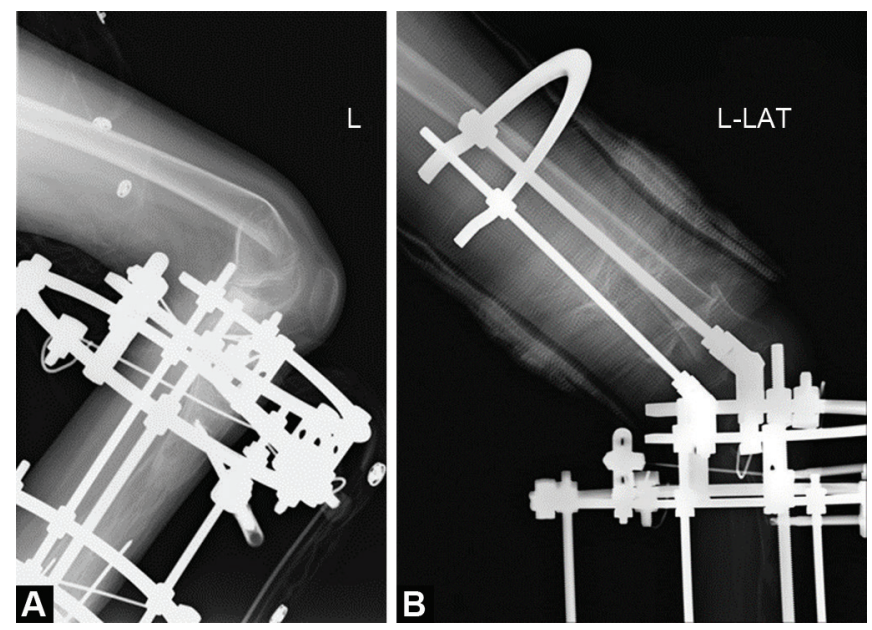

Figs $3 \mathrm{~A}$ and $\mathrm{B}$ : Case 1: Plain radiograph taken at the time of injury showing a left femoral supracondylar greenstick fracture treated via closed reduction and stabilisation
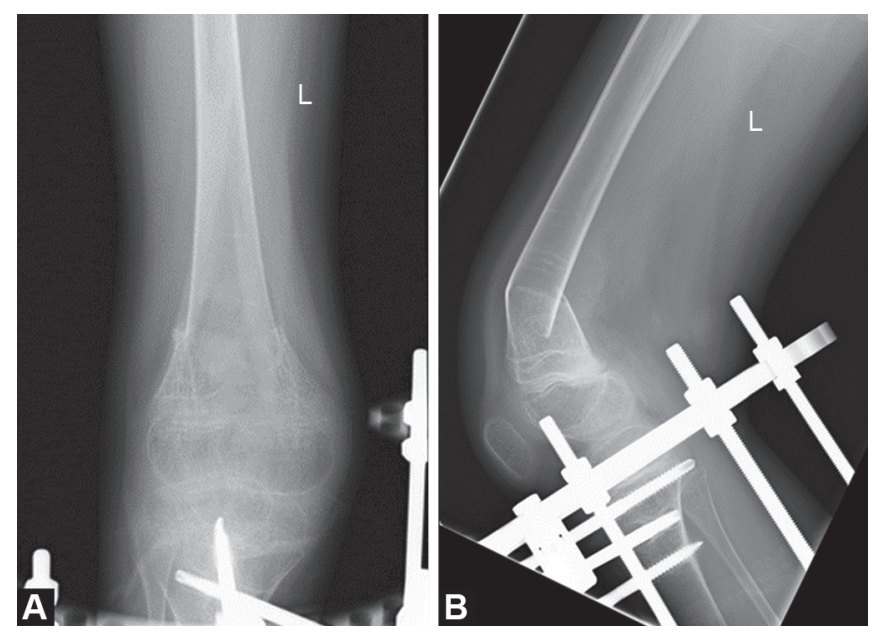

Figs $5 \mathrm{~A}$ and B: Case 2: Plain radiograph taken at the time of injury showing a right femoral supracondylar fracture

fractures secondary to half-pin usage in adults have been reported. One study ${ }^{8}$ reported that stress fractures had occurred in 10 of 254 patients being treated for Charcot foot and in 3 patients after the application of an additional circular external fixator.

Limb lengthening and reconstruction methods, such as bone transport, take a long time for the maturation of the regenerated bone. Children with a circular external fixator during such treatment are encouraged to bear weight to promote such maturation. ${ }^{15}$

All three patients in the present series had femoral supracondylar greenstick fractures. The mechanism of this injury seems to be the restriction of knee flexion due to the frame itself. In addition, the restriction of ankle motion leads to gait disturbance, loss of balance, and increases the risk of falling. During a fall, the stress is concentrated in the distal femoral metaphysis, which results in a femoral supracondylar fracture.

A periprosthetic distal femoral fracture after a total knee arthroplasty is also relatively common; some studies have reported satisfactory outcomes for conservative treatment of such fractures. ${ }^{16,17}$ Nasr et al. ${ }^{18}$ compared the outcomes of conservative and surgical management of adult supracondylar femoral fractures and concluded that conservative management tends to lead a poor

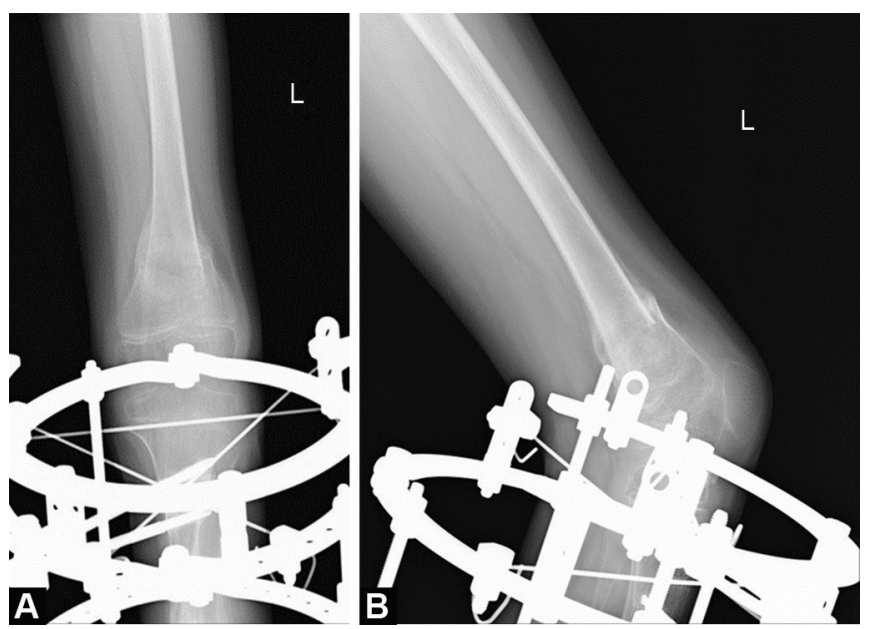

Figs $4 \mathrm{~A}$ and $\mathrm{B}$ : Solid union is achieved with no complications

functional result. Thus, adult supracondylar femoral fractures are treated surgically. A systematic review showed that periprosthetic distal femoral fractures are most successfully treated with a locking plate or intramedullary nail, and that the complication rate for locked plating is lower than that for intramedullary nailing. ${ }^{10}$

Paediatric supracondylar femoral fractures represent $12 \%$ of all paediatric femoral fractures. However, the treatment strategy for this fracture in children differs from that in adults. Paediatric fractures are often treated conservatively by casting or splinting because these fractures heal more quickly than adult fractures and children have a high capacity for remodelling. ${ }^{19}$ Several treatment methods have been used, such as skeletal traction, casting, percutaneous K-wire fixation, ${ }^{11}$ titanium elastic nailing, ${ }^{12}$ and locked plating. ${ }^{14}$ These supracondylar femoral fractures have been treated by closed reduction with percutaneous K-wire fixation and cast augmentation in young children, but this strategy may not provide adequate stability in larger children and is associated with some complications. ${ }^{20}$ The overall incidence of refracture after external fixation for paediatric femoral fractures is estimated to be as high as $12 \%{ }^{21}$ to $30 \% .^{22}$

Fixation of a supracondylar femoral fracture by casting or splinting if the patient has a circular external fixator on the ipsilateral tibia is fraught with difficulty. One study reported the pin and plaster technique, ${ }^{11}$ whereas another study reported use of an external fixator, but this resulted in complications. ${ }^{13}$ Furthermore, despite being percutaneous, both are still invasive procedures. Titanium elastic nailing has also been used, ${ }^{12,23}$ while plate internal fixation is more invasive and can carry a risk of physeal damage, depending on the fracture type. ${ }^{14,20}$

The novel hybrid fixation technique described in this study is a conservative treatment that does not require invasive surgery. Moreover, no special technique is required. Reduction is confirmed and maintained by fluoroscopy, and the knee position can be adjusted using the lateral hinges. Early knee range of motion exercise can be started after confirmation of fracture stability. This technique also prevents development of knee contractures and promotes early recovery.

The limitations of this novel hybrid fixation technique are that it is unclear whether it can be applied in large children over 12 years old who take a longer time to heal, or in patients with very unstable fractures. 

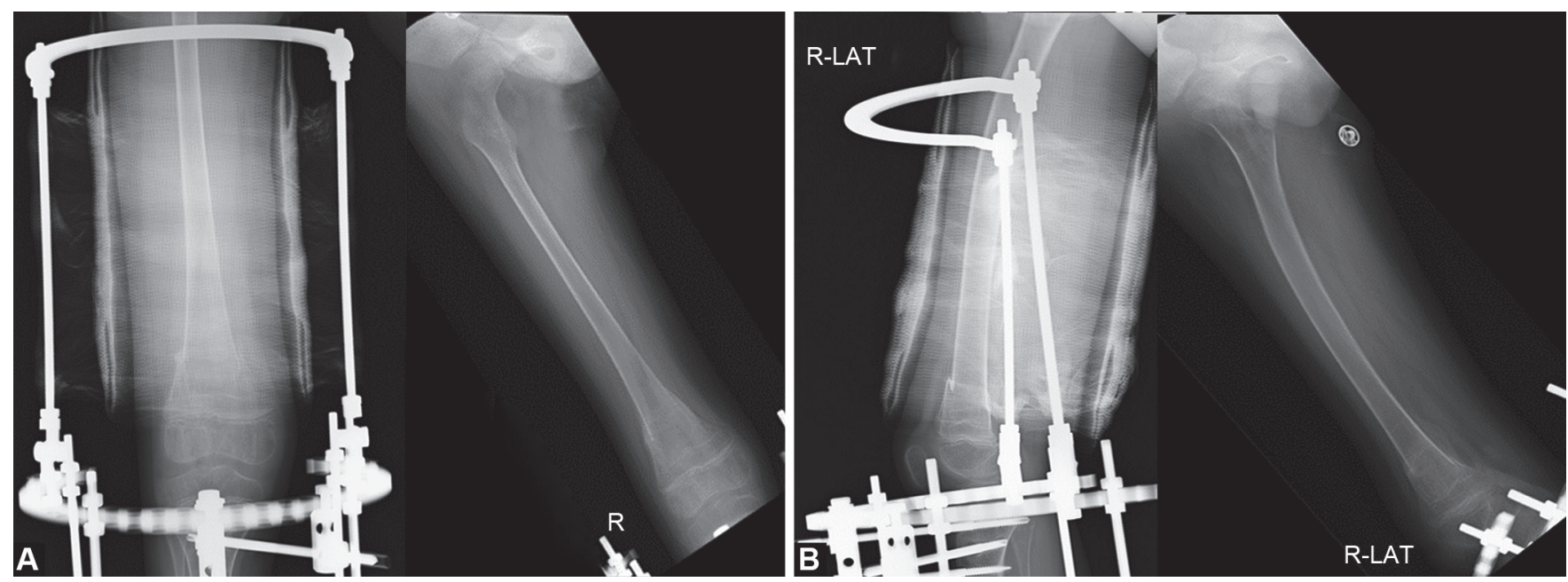

Figs 6A and B: Case 2: The right femoral supracondylar fracture is stabilised by hybrid fixation, with removal of the cast and rods after solid union is achieved at 21 days

\section{Conclusion}

A hybrid fixation technique for paediatric supracondylar femoral fractures is described for children who have circular external fixation on the tibia. This treatment has led to no complications, with an early return to exercise and recovery. The hybrid fixation technique is suited for younger patients and is minimally invasive, easy to perform, and provides adequate stability and a reliable outcome.

\section{Additional Notes}

We thank Kelly Zammit, BVSc, from Edanz Editing (www. edanzediting.com/ac), for editing a draft of this manuscript.

\section{Ethics Approval}

All procedures performed in this study involving human participants were in accordance with the ethical standards of the institutional and with the 1964 Helsinki declaration and its later amendments or comparable ethical standards.

\section{Consent to Participate}

Written informed consent was obtained from the patients' guardians.

\section{Consent for Publication}

The patients' guardians provided written informed consent for the publication this study. The images are completely anonymous, and the text does not carry any identifying information.

\section{References}

1. Tafazal S, Madan SS, Ali F, et al. Management of paediatric tibial fractures using two types of circular external fixator: Taylor spatial frame and Ilizarov circular fixator. J Child Orthop 2014;8(3):273-279. DOI: 10.1007/s11832-014-0583-2.

2. Zenios M, Oyadiji SO. Effect of asymmetrical configuration of pins in the TSF external fixator used for tibial lengthening in a pediatric population. J Pediatr Orthop 2014;34(6):618-624. DOI: 10.1097/ BPO.0000000000000194.

3. Demiralp B, Ege T, Kose O, et al. Reconstruction of intercalary bone defects following bone tumor resection with segmental bone transport using an Ilizarov circular external fixator. J Orthop Sci 2014;19(6):1004-1011. DOI: 10.1007/s00776-014-0632-1.

4. Paley D. Problems, obstacles, and complications of limb lengthening by the llizarov technique. Clin Orthop Relat Res 1990;250(250):81-104. DOI: 10.1097/00003086-199001000-00011.

5. De Bastiani G, Aldegheri R, Renzi-Brivio L, et al. Limb lengthening by callus distraction (callotasis). J Pediatr Orthop 1987;7(2):129-134. DOI: 10.1097/01241398-198703000-00002.

6. Simpson $\mathrm{AH}$, Kenwright J. Fracture after distraction osteogenesis. J Bone Joint Surg Br 2000;82(5):659-665. DOI: 10.1302/0301620x.82b5.9945.

7. Launay $F$, Younsi $R$, Pithioux $M$, et al. . Fracture following lower limb lengthening in children: a series of 58 patients. Orthop Traumatol Surg Res 2013;99(1):72-79. DOI: 10.1016/j.otsr.2012.08.005.

8. Jones $\mathrm{CP}$, Youngblood CSA, Waldrop N, et al. Tibial stress fracture secondary to half-pins in circular ring external fixation for Charcot foot. Foot Ankle Int 2014;35(6):572-577. DOI: 10.1177/1071100714531229.

9. Blair JA, Owens JG, Saucedo J, et al. Skeletal trauma research consortium (STReC). Functional rehabilitation with a foot plate modification for circular external fixation. Foot Ankle Int 2013;34(6):890-897. DOI: 10.1177/1071100713483975.

10. Ebraheim NA, Kelley LH, Liu X, et al. Periprosthetic distal femur fracture after total knee arthroplasty: a systematic review. Orthop Surg 2015;7(4):297-305. DOI: 10.1111/os.12199.

11. Memisoglu K, Atmaca H, Kesemenli CC. Treatment of femur fractures in preschool children with double pin technique: immediate incorporated hip spica casting by two K-wires. Indian J Surg 2015;77(Suppl 2):635-639. DOI: 10.1007/s12262-013-0952-y.

12. Parikh SN, Nathan ST, Priola MJ, et al. Elastic nailing for pediatric subtrochanteric and supracondylar femur fractures. Clin Orthop Relat Res 2014;472(9):2735-2744. DOI: 10.1007/s11999-013-3240-z.

13. Sabharwal S. Role of Ilizarov external fixator in the management of proximal/distal metadiaphyseal pediatric femur fractures. J Orthop Trauma 2005;19(8):563-569.DOI: 10.1097/01.bot.0000174706.03357.26.

14. Abdelgawad AA, Kanlic EM. Pediatric distal femur fixation by proximal humeral plate. J Knee Surg 2013;26(Suppl 1):S45-S49. DOI: 10.1055/s0031-1299658.

15. Hohmann E, Birkholtz F, Glatt V, et al. The "Road to Union" protocol for the reconstruction of isolated complex high-energy tibial trauma. Injury 2017;48(6):1211-1216. DOI: 10.1016/j.injury.2017.03.018.

16. Delport PH, Van Audekercke R, Martens M, et al. Conservative treatment of ipsilateral supracondylar femoral fracture after total knee arthroplasty. J Trauma 1984;24(9):846-849. DOI: 10.1097/00005373-198409000-00013.

17. Sochart $\mathrm{DH}$, Hardinge K. Nonsurgical management of supracondylar fracture above total knee arthroplasty. Still the nineties 
option. J Arthroplasty 1997;12(7):830-834. DOI: 10.1016/s08835403(97)90016-4.

18. Nasr AM, Mc Leod I, Sabboubeh A, et al. Conservative or surgical management of distal femoral fractures. A retrospective study with a minimum five year follow-up. Acta Orthop Belg 2000;66(5):477-483.

19. Rickert KD, Hosseinzadeh $P$, Edmonds EW. What's new in pediatric orthopaedic trauma: the lower extremity. J Pediatr Orthop 2018;38(8):e434-e439. DOI: 10.1097/BPO.0000000000001209.

20. Bor N, Rozen N, Dujovny E, et al. Fixator-assisted plating in pediatric supracondylar femur fractures. Glob Pediatr Health 2019;6:2333794X19843922. DOI: 10.1177/2333794X19843922.
21. Skaggs DL, Leet Al, Money MD, et al. Secondary fractures associated with external fixation in pediatric femur fractures. J Pediatr Orthop 1999;19(5):582-586. DOI: 10.1097/01241398-19990900000005.

22. Gregory P, Pevny T, Teague D. Early complications with external fixation of pediatric femoral shaft fractures. J Orthop Trauma 1996;10(3):191-198. DOI: 10.1097/00005131-19960400000007.

23. Smith NC, Parker D, McNicol D. Supracondylar fractures of the femur in children. J Pediatr Orthop 2001;21(5):600-603. DOI: 10.1097/01241398200109000-00009. 